\title{
A theory of magnetization reversal in nanowires
}

\author{
Robert S. Maier \\ Depts. of Mathematics and Physics, University of Arizona, Tucson, AZ 85721, USA
}

\begin{abstract}
Magnetization reversal in a ferromagnetic nanowire which is much narrower than the exchange length is believed to be accomplished through the thermally activated growth of a spatially localized nucleus, which initially occupies a small fraction of the total volume. To date, the most detailed theoretical treatments of reversal as a field-induced but noise-activated process have focused on the case of a very long ferromagnetic nanowire, i.e., a highly elongated cylindrical particle, and have yielded a reversal rate per unit length, due to an underlying assumption that the nucleus may form anywhere along the wire. But in a bounded-length (though long) cylindrical particle with flat ends, it is energetically favored for nucleation to begin at either end. We indicate how to compute analytically the energy of the critical nucleus associated with either end, i.e., the activation barrier to magnetization reversal, which governs the reversal rate in the low-temperature (Kramers) limit. Our treatment employs elliptic functions, and is partly analytic rather than numerical. We also comment on the Kramers prefactor, which for this reversal pathway does not scale linearly as the particle length increases, and tends to a constant in the low-temperature limit.
\end{abstract}

Keywords: Kramers theory, weak spatiotemporal noise, Landau-Lifshitz-Gilbert equation, activation barrier, Kramers prefactor, ferromagnetic cylinder, ferromagnetic nanowire, magnetization reversal, Néel-Brown theory

\section{INTRODUCTION}

The phenomenon of magnetization reversal is of intense interest to the data storage and recording industries, for obvious reasons. Most treatments of this phenomenon have employed the standard techniques of micromagnetic modeling: analytically or numerically solving a partial differential equation satisfied by the magnetization vector, which is valid in an averaged, coarse-grained sense, on scales large compared to the crystal lattice [1]. Ferromagnetic samples of many geometries have been studied theoretically [2].

Provided that a sufficiently strong reversed field is applied to a ferromagnetic sample, the reversal of the direction of magnetization is an essentially deterministic process, which if the sample is sufficiently large (larger than the coherence length) will begin with the formation of a spatially localized 'nucleus' of reversed magnetization. This nucleus will grow, eventually yielding a global reversal of the magnetization. This is in contrast to the case when the applied field is less strong than the nucleation field, in which a critical nucleus can only be formed by thermal fluctuations, though its subsequent growth may be deterministic. The two types of reversal are sometimes called field-activated and noise-activated. In many applications, such as quantifying the likelihood of loss of stored data, it is the second phenomenon, more closely tied to statistical mechanics than to the theory of deterministic evolution equations, which is of interest.

The difference between the two types of reversal is made clear by an analogy. Consider an oversimplified overdamped scalar model of the form $\dot{m}=-U^{\prime}(m)+$ noise, where $U(\cdot)$ is a bistable effective potential, the two minima of which correspond to an alignment of the magnetization with and against an applied field. As the field strengthens, this double-well potential may become sufficiently skewed that the latter of the two minima disappears, leading to a rapid fall into the remaining minimum, which corresponds to alignment with the field. This is the essence of field-activated nucleation. On the other hand, if the field is insufficiently strong, then there will remain two minima: a metastable one (against the field) and a stable one (with the field). It is clear that noise-induced nucleation is in essence a standard problem of noise-induced escape from a metastable state. Of course, a satisfactory model will need to be more sophisticated than this scalar model. Even if the

Further author information:

E-mail: rsm@math.arizona.edu, Telephone: +15206212671 
sample is small ("zero-dimensional"), so that reversal takes place by a process of spatially coherent rotation of the magnetization vector, the vectorial nature of the magnetization is crucial.

The process of noise-activated reversal by coherent rotation was first treated theoretically by Brown $[3$, who by adapting the Kramers theory of weak-noise escape rates was able to work out an asymptotic (low-temperature) expression for the reversal rate in a single-domain particle with uniaxial anisotropy. His work has been extended to the case of cubic anisotropy. However, the issue of which reversal mechanisms are dominant in large ferromagnetic particles remains controversial. The simplest sort of non-zero-dimensional sample is a ferromagnetic nanowire: a long, thin cylinder of width at most a few tens of nanometers. The most extensive treatment of field-induced but noise-activated magnetization reversal in such a sample is that of Braun [4, 5, 6, 7, 8, By applying the infinitedimensional Kramers theory of Langer and Coleman [9] to a stochastically perturbed Landau-Lifshitz-Gilbert equation governing the evolution of the magnetization vector along the nanowire, he worked out analytically an asymptotic expression for the weak-noise reversal rate. However, his treatment assumes a toroidal sample (with periodic boundary conditions), and applies only in the limit of infinite sample length. In this limit, the nucleus of his model is a metastable field configuration of soliton type, with a simple functional form. It comprises a 'critical droplet' of reversed magnetization bounded by a $\pi$ wall and a $-\pi$ wall; this droplet may form with equal likelihood anywhere along the sample. His computed reversal rate is accordingly proportional to the sample length $L$. It also contains a factor $\exp \left(-\Delta E / k_{B} T\right)$, where $\Delta E$ is the energy of formation of the droplet. Like Brown's asymptotic reversal rate formula, Braun's contains corrections to the Arrhenius (i.e., exponential) low-temperature falloff. For reasons explained below, both fall off as $T^{-1 / 2} \exp \left(-\Delta E / k_{B} T\right)$ as $T \rightarrow 0$. A nonArrhenius behavior of the reversal rate as $T \rightarrow 0$ has been found in stochastic simulations of single-domain particles, at least [10].

Aharoni [1] 12] has criticized Braun's not including the (nonlocal) magnetostatic self-energy in his LandauLifshitz-Gilbert Hamiltonian. If the magnetization is not solenoidal $(\boldsymbol{\nabla} \cdot \mathbf{m} \neq 0)$, there will be a magnetostatic volume charge, which will generate a demagnetizing field. This term can be neglected in a planar topology [13, but not in the cylindrical topology. Aharoni has also criticized the assumption that the magnetization vector is constant across the cross-section of the cylindrical sample, which prevents reversal from occurring via a 'curling' mode. However, this assumption is presumably justified in the limit of small sample width. Also, by what amounts to dimensional analysis, Braun has shown that in this limit the nonlocal part of the selfenergy is relatively small, and the local part may be absorbed into a renormalization of crystalline anisotropy constants [14. With these emendations, Braun's analysis seems to be applicable to thin cylindrical samples of at least some materials, though of course the assumption of a very long sample with periodic boundary conditions is unrealistic and will need to be dropped.

In this extended abstract we indicate how the analytic treatment of Braun can be extended to the case of a thin cylinder with flat ends, at which open (i.e., Neumann) boundary conditions on the magnetization vector are appropriate. We do this by adapting our work on reversal in a real Ginzburg-Landau equation perturbed by weak spatiotemporal noise [15]. Since we do not take the $L \rightarrow \infty$ limit, our expression for the metastable nucleus configuration is more complicated than Braun's. It involves elliptic functions [16, which have long been used in the analytic modeling of periodic micromagnetic structures. (See ref. [17, §2.4, and ref. [18]) Sophisticated numerical simulations at the magnetic moment level of noise-activated reversal, in a thin cylinder with flat ends, show that a droplet of reversed magnetization forms at one of the ends and spreads inward [19] 20. This reversal mode is in agreement with our analytic treatment. We also comment on the prefactor in the low-temperature reversal rate, i.e., the factor that multiplies $\exp \left(-\Delta E / k_{B} T\right)$. As $L \rightarrow \infty$, it does not grow linearly in $L$, but rather tends to a constant, since the formation of a droplet of reversed magnetization away from both ends of the cylinder is energetically disfavored. We also find that the prefactor includes no $T^{-1 / 2}$ factor. That is, the rate of noise-activated magnetization reversal, if physically reasonable boundary conditions are imposed, follows an Arrhenius law.

\section{ESCAPE FROM A METASTABLE STATE}

The theory of noise-activated fluctuations away from a metastable state [21, including escape to a stable state, was developed most significantly by Kramers [22]. Major contributions were also made by Eyring, who worked on escape in multidimensional state spaces in the context of reaction-rate theory. It should be emphasized that 
the Kramers approach to escape rates is asymptotic: increasingly accurate in the weak-noise limit, in which escape per unit time becomes exponentially unlikely. In this limit, the phenomenology of noise-activated escape from the domain of attraction of a metastable state involves eventual motion along an 'optimal trajectory' that typically goes uphill from the metastable state to a saddle point, near which exit occurs. By definition, this is the escape pathway of least resistance. The escape rate is computed as the integral of the outgoing probability flux through a region on the boundary of the domain of attraction which is centered on the saddle point. The optimal trajectory picture and the associated computations become increasingly valid in the weak-noise limit.

The present author and his collaborators have extended Kramers theory to the case when the system being modeled, if noise is absent, has 'nongradient' dynamics: for example, when its order parameter $\mathbf{x}$ (not a magnetization, in general) is finite-dimensional but does not evolve according to any law of the form $\dot{\mathbf{x}}=-\nabla U(\mathbf{x})$. This could be because the dynamics include dissipation or non-conservative forces. In the nongradient case the phenomenology of escape may be quite different from that described above. The domain of attraction of a metastable state may have an unstable limit cycle as its boundary, and the outgoing optimal trajectory may spiral into this limit cycle. This is in fact a feature of the abovementioned coherent rotation model of Brown [3], which is Hamiltonian but includes a damping term. In two-dimensional models in which the boundary of the domain of attraction is an unstable limit cycle, the Kramers escape rate must be computed as the integral of the outgoing probability flux over the entire boundary, rather than merely over the vicinity of a saddle point (which may not be present). This usually gives rise to anomalous weak-noise behavior of the Kramers prefactor, i.e., the factor that multiplies the usual exponential suppression factor in the asymptotic weak-noise escape rate.

In this section we review the extensions to Kramers rate theory that we shall need in understanding and extending the results of Braun [4, 5, 6, 7, 8, with an emphasis on the prefactor and on the phenomenology of escape. We shall use dimensionless units throughout. Suppose a point $\mathbf{x}$ in finite-dimensional Euclidean space (or possibly on a surface such as a sphere) evolves according to the overdamped Langevin equation

$$
\dot{\mathbf{x}}=\mathbf{u}(\mathbf{x})+\epsilon^{1 / 2} \boldsymbol{\eta}(t) .
$$

Here $\mathbf{u}$ is a drift field, $\boldsymbol{\eta}$ is normalized white noise, which satisfies

$$
\left\langle\eta_{i}\left(t_{1}\right) \eta_{j}\left(t_{2}\right)\right\rangle=\delta_{i j} \delta\left(t_{1}-t_{2}\right),
$$

and $\epsilon \ll 1$ is a noise-strength parameter. Suppose the drift field has a metastable point $S$ and a stable point $S^{\prime}$, with a saddle point denoted $U$ (for unstable) between them, on the common boundary of their domains of attraction. If $\mathbf{x}=S$ initially, the time for noise-activated escape can be defined as the time needed for $\mathbf{x}$ to reach some specified region $R$ that lies strictly within the domain of attraction of $S^{\prime}$, and contains $S^{\prime}$ itself. This escape time $t_{\text {exit }}\left(S \rightarrow S^{\prime}\right)$ is a random variable, the distribution of which in the weak-noise limit will not be much affected by the choice of region $R$. As $\epsilon \rightarrow 0$, its mean $\left\langle t_{\text {exit }}\left(S \rightarrow S^{\prime}\right)\right\rangle$ will grow exponentially (to leading order), and its distribution will increasingly resemble that of an exponential random variable. The escape rate $\Gamma_{S \rightarrow S^{\prime}}$ can be defined as the reciprocal $\left\langle t_{\text {exit }}\left(S \rightarrow S^{\prime}\right)\right\rangle^{-1}$. Just as there is noise-activated motion from $S$ to $S^{\prime}$, there is noise-activated motion from $S^{\prime}$ back to $S$, but reverse motion is exponentially suppressed in the weak-noise limit, relatively as well as absolutely.

The escape rate $\Gamma_{S \rightarrow S^{\prime}}$ may be written as $\Gamma^{0} \exp (-\Delta W / \epsilon)$, where $\Delta W$ is the minimum amount of energy the noise must put into the system to drive it over the saddle $U$. By definition, the second factor is the Kramers factor, and $\Gamma^{0}$ is the Kramers prefactor. The case when the drift field $\mathbf{u}$ is the negative gradient of a potential function, i.e., $\mathbf{u}=-\nabla V$, is the easiest case to treat, since the optimal escape trajectory is simply a time-reversed relaxational trajectory, and satisfies $\dot{\mathbf{x}}=-\mathbf{u}(\mathbf{x})$. It turns out that $W=2 V$ (the factor of 2 is sometimes absorbed into a redefinition of $\epsilon$ ). The weak-noise behavior of the escape rate can readily be expressed in terms of linear approximations to the drift field at $\mathbf{x}=S$ and $\mathbf{x}=U$, by the Kramers-Eyring formula. In the two-dimensional case, this formula is

$$
\Gamma_{S \rightarrow S^{\prime}} \sim \frac{1}{2 \pi} \sqrt{\left|\lambda_{\|}(U)\right| \lambda_{\|}(S)} \sqrt{\frac{\lambda_{\perp}(S)}{\lambda_{\perp}(U)}} \exp (-2 \Delta V / \epsilon), \quad \epsilon \rightarrow 0 .
$$

Here $\lambda_{\|}(S) \equiv-\partial u_{S, \|} / \partial x_{S, \|}$ is the eigenvalue of the linearized negative drift field at $S$ whose corresponding eigenvector points along $\hat{\mathbf{x}}_{S, \|}$, the direction locally parallel to the departing optimal trajectory. Similarly, 
$\lambda_{\perp}(U) \equiv-\partial u_{U, \perp} / \partial x_{U, \perp}$ is the eigenvalue of the linearized negative drift field at $U$ whose corresponding eigenvector points along $\hat{\mathbf{x}}_{U, \perp}$, the direction locally perpendicular to the approaching optimal trajectory, and so forth. $\lambda_{\|}(U)$ is the eigenvalue of the linearized negative drift field at $U$ that corresponds to the unstable, or 'downhill' direction; it is negative. The formula (3) generalizes immediately to the case when the order parameter $\mathbf{x}$ has arbitrary finite dimensionality $d$. In general, the prefactor will contain a product of $d-1$ factors resembling $\sqrt{\frac{\lambda_{\perp}(S)}{\lambda_{\perp}(U)}}$, one for each direction transverse to the optimal trajectory.

The standard formula (3) contains a constant prefactor, which does not include any power of the noise strength $\epsilon$. But the derivation of (3) makes clear how such a power could arise. The stationary probability density function (PDF) of the order parameter $\mathbf{x}$ is $\rho_{0}(\mathbf{x}) \equiv \exp (-W(\mathbf{x}) / \epsilon)$, up to normalization. The slowest decaying nonstationary mode of the drift-diffusion equation for the PDF is a quasistationary PDF $\rho_{1}$, which characterizes how probability equilibrates between the domains of attraction of $S$ and $S^{\prime}$. Like $\rho_{0}$, it will contain an $\exp (-2 V(\mathbf{x}) / \epsilon)$ factor, so it will typically be concentrated on the $O\left(\epsilon^{1 / 2}\right)$ transverse lengthscale near the saddle point $U$. But unlike $\rho_{0}, \rho_{1}$ will have opposite signs in the two domains. Along the $\hat{\mathbf{x}}_{U, \|}$ direction through $U$, it will change sign. The rate $\Gamma_{S \rightarrow S^{\prime}}$ is the rate at which probability diffuses across the saddle, from $S$ toward $S^{\prime}$. As Kramers showed, this is easily evaluated as an integral of the probability flux. In this integral there are several powers of $\epsilon$ that must be kept track of. For example, there is a normalization factor proportional to $\epsilon^{-d / 2}$ in $\rho_{1}$ (as there is in $\rho_{0}$ ). Also, it follows from the Langevin equation (11) that besides drifting along $\mathbf{u}$, the PDF diffuses with diffusivity $\epsilon$. A careful evaluation of the probability flux integral yields the Kramers prefactor shown in (31), with all powers of $\epsilon$ canceling.

A prefactor containing a nontrivial power of $\epsilon$ could arise from the potential energy $V=V(\mathbf{x})$ behaving nonquadratically near $\mathrm{x}=S$ or $U$, causing one or more of the four eigenvalues appearing in (3) to equal zero [23]. As mentioned, non-Arrhenius behavior will also arise in the case of a two-dimensional order parameter if the separatrix between the domains of attraction of $S$ and $S^{\prime}$ contains no saddle point, and is an unstable limit cycle. For this to be the case, the drift $\mathbf{u}$ cannot be the negative gradient of a potential function. A transverse integration of the probability flux generated by the quasistationary PDF $\rho_{1}$ over an $O(1)$ lengthscale around a limit cycle, rather than over an $O\left(\epsilon^{1 / 2}\right)$ transverse lengthscale near a saddle point, will yield an escape rate larger by a factor roughly proportional to $\epsilon^{1 / 2}$. In such a situation,

$$
\Gamma_{S \rightarrow S^{\prime}} \sim \text { const } \times \epsilon^{-1 / 2} \exp (-\Delta W / \epsilon), \quad \epsilon \rightarrow 0,
$$

where 'const' can be evaluated as an integral around the separatrix, will replace (31).

All of the preceding remarks apply to the case of finite-dimensional overdamped dynamics, where the Langevin equation for the order parameter has the form (11). If there is damping but the strength of the damping is not taken to infinity, a more appropriate Langevin equation in dimensionless units is

$$
\ddot{\mathbf{x}}+\alpha \dot{\mathbf{x}}=\mathbf{u}(\mathbf{x})+\epsilon^{1 / 2} \boldsymbol{\eta}(t),
$$

where $\alpha>0$ is a damping constant, or equivalently

$$
\left\{\begin{aligned}
\dot{\mathbf{x}} & =\mathbf{v} \\
\dot{\mathbf{v}} & =-\alpha \mathbf{v}+\mathbf{u}(\mathbf{x})+\epsilon^{1 / 2} \boldsymbol{\eta}(t),
\end{aligned}\right.
$$

where $\mathbf{v} \equiv \dot{\mathbf{x}}$. This is effectively an overdamped Langevin equation for a $2 d$-dimensional order parameter $(\mathbf{x}, \mathbf{v})$ that diffuses only in the velocity direction. But if $\alpha$ has some fixed positive value, the weak-noise behavior of the Kramers prefactor in this non-overdamped model is surprisingly similar to that discussed above. The prefactor becomes $\alpha$-dependent [21], but there is still no $\epsilon$-dependence if $\mathbf{u}=-\nabla V$ with $V$ quadratic near $S$ and $U$. When $d=2$, an $\epsilon^{-1 / 2}$ factor will appear in the Kramers prefactor if $\mathbf{u}$ is not derived from a potential and there is no saddle on the separatrix, for essentially the reason already discussed. There are clearly some subtleties associated with the reduction of the non-overdamped model of (15) to the overdamped model of (11) when $\alpha \rightarrow \infty$, but we do not discuss them here.

Infinite-dimensional Kramers theory is a theory of rate and relaxation phenomena exhibited by random fields, rather than by randomly evolving finite-dimensional order parameters. However, it is formally quite similar to 
finite-dimensional Kramers theory. This is perhaps not entirely clear from standard expositions [9], but we shall make it so. For simplicity, consider a field $\phi=\phi(x, t)$ perturbed by noise of strength $\epsilon$, which evolves with overdamped dynamics on a spatial domain $[0, L]$ according to

$$
\dot{\phi}=-\delta \mathcal{H} / \delta \phi+\epsilon^{1 / 2} \eta(x, t) .
$$

The first term on the right-hand side is the functional derivative of an energy functional, and $\eta(x, t)$ is normalized spatiotemporal white noise, which satisfies

$$
\left\langle\eta\left(x_{1}, t_{1}\right) \eta\left(x_{2}, t_{2}\right)\right\rangle=\delta\left(x_{1}-x_{2}\right) \delta\left(t_{1}-t_{2}\right),
$$

i.e., has a flat power spectrum in space as well as time. Typically

$$
\mathcal{H}[\phi]=\int_{0}^{L}\left[\frac{\phi^{\prime}(x)^{2}}{2}+V(\phi(x))\right] d x,
$$

so that the evolution equation is a stochastically perturbed Ginzburg-Landau equation, namely

$$
\dot{\phi}=\Delta \phi-V^{\prime}(\phi)+\epsilon^{1 / 2} \eta(x, t) .
$$

If the potential $V$ is bistable or multistable then noise-induced transitions between stable spatially extended states, each of which is concentrated around a field value that is a minimum of $V$, may occasionally take place. Each such transition will involve a passage through an intermediate (unstable) saddle state.

These spatially extended states are strongly affected by the boundary conditions at $x=0$ and $x=L$. Suppose that $V(\phi)$ has two local minima, at $\phi=S, S^{\prime}$, with the latter being the global minimizer; and that the intervening local maximum is at $\phi=U$. If periodic or Neumann boundary conditions are imposed, one would expect that the metastable, saddle, and stable states are the constant field configurations $\phi \equiv S, U, S^{\prime}$; though if (say) Dirichlet boundary conditions are imposed, one would expect them to differ, especially near $x=0$ and $x=L$. Actually, even in the periodic and Neumann cases the saddle state most relevant to noise-induced transitions may not be the constant solution $\phi \equiv U$. If $L$ is sufficiently large, there may be a lower-energy saddle in the field configuration space, which is spatially oscillatory rather than constant [15]. The importance of such nonconstant saddle configurations was first pointed out by Langer 24] in a study of the $L \rightarrow \infty$ limit of escape from a metastable state. Due to their oscillatory character they are sometimes called "Langer's bounces". Each such oscillatory saddle configuration can be viewed as a background metastable $(\phi \equiv S)$ state, within which a droplet of the stable $\left(\phi \equiv S^{\prime}\right)$ state has formed. The droplet is critical, which means that if pushed in the right direction, the saddle configuration may roll 'downhill' to the stable rather than the original metastable state.

The Kramers-Eyring formula (3) generalizes readily to the infinite-dimensional case, becoming

$$
\Gamma \sim \frac{\left|\lambda_{0}\left(\phi_{u}\right)\right|}{2 \pi} \sqrt{\frac{\prod_{n=0}^{\infty} \lambda_{n}\left(\phi_{s}\right)}{\prod_{n=0}^{\infty}\left|\lambda_{n}\left(\phi_{u}\right)\right|}} \exp (-2 \Delta \mathcal{H} / \epsilon), \quad \epsilon \rightarrow 0 .
$$

Here $\left\{\lambda_{n}\left(\phi_{s}\right)\right\}_{n=0}^{\infty}$ and $\left\{\lambda_{n}\left(\phi_{u}\right)\right\}_{n=0}^{\infty}$ are the eigenvalues of the linearizations of the negative drift field $\delta \mathcal{H} / \delta \phi$ at the metastable state $\phi=\phi_{s}(x)$ and the dominant saddle $\phi=\phi_{u}(x)$, arranged in increasing order. The only negative eigenvalue in either set is $\lambda_{0}\left(\phi_{u}\right)$, which corresponds to 'downhill' motion away from the saddle. It is easy to see that in the two-dimensional case, (11) reduces to (3).

Because the numerator and denominator of the quotient inside the square root are products of an infinite number of eigenvalues, which tend to infinity as $n \rightarrow \infty$, each diverges. However, their quotient can be defined as a limit of finite-dimensional truncations. Usually it is recast as a determinant quotient, computed from the linearized deterministic evolution operators at $\phi=\phi_{s}, \phi_{u}$ [25] 26]. Consider for example a small perturbation $\xi$ away from the metastable state, i.e., $\phi=\phi_{s}+\xi$. Then to leading order $\dot{\xi}=-\hat{\Lambda}\left[\phi_{s}\right] \xi$, where $\hat{\Lambda}\left[\phi_{s}\right] \equiv \delta^{2} \mathcal{H} / \delta \phi^{2}\left[\phi_{s}\right]$ specifies the linearized zero-noise dynamics near $\phi=\phi_{s}$. Similarly, $\hat{\Lambda}\left[\phi_{u}\right]$ specifies the dynamics near $\phi=\phi_{u}$. For the model defined by (8), $\hat{\Lambda}\left[\phi_{s}\right], \hat{\Lambda}\left[\phi_{u}\right]$ equal $-\Delta+V^{\prime \prime}\left(\phi_{s}(x)\right),-\Delta+V^{\prime \prime}\left(\phi_{u}(x)\right)$. One can write

$$
\Gamma_{\phi_{s} \rightarrow \phi_{s^{\prime}}} \sim \frac{\left|\lambda_{0}\left(\phi_{u}\right)\right|}{2 \pi} \sqrt{\frac{\operatorname{det} \hat{\Lambda}\left[\phi_{s}\right]}{\left|\operatorname{det} \hat{\Lambda}\left[\phi_{u}\right]\right|}} \exp (-2 \Delta \mathcal{H} / \epsilon), \quad \epsilon \rightarrow 0 .
$$


The two determinants of Schrödinger operators are not defined individually, but their quotient can be made sense of by any of several regularization schemes; for example, zeta-function and dimensional regularization [26].

If the boundary conditions at $x=0, L$ are periodic, so that the spatial domain $[0, L]$ is effectively a circle, and the dominant (lowest-energy) saddle state $\phi=\phi_{u}(x)$ is not the constant solution $\phi \equiv U$ but rather a field configuration containing a critical droplet, the asymptotic formula (12) must be modified. Since the droplet configuration can be shifted arbitrarily around $[0, L]$, the operator $\hat{\Lambda}\left[\phi_{u}\right]$ will have a zero eigenvalue $\lambda_{1}\left(\phi_{u}\right)$, the accompanying eigenfunction being a Goldstone mode that expresses translation invariance. So in this case the prefactor of (12) will diverge, signalling a change in the phenomenology of escape. In the infinite-dimensional space of field configurations, the separatrix between the domains of attraction of the metastable and stable states will now include an unstable limit cycle of length $L$, and noise-activated escape may take place anywhere along it. We have already explained how to handle such separatrices. Since the escape rate must be computed by integrating the outgoing probability flux around the separatrix, rather than on a transverse lengthscale of magnitude $O\left(\epsilon^{1 / 2}\right)$, the prefactor will be larger by a factor that grows like $L \epsilon^{-1 / 2}$ as $L \rightarrow \infty$. The asymptotic weak-noise behavior of the escape rate in this case, when $L$ is fixed, is accordingly of the form

$$
\begin{array}{lll}
\Gamma_{\phi_{s} \rightarrow \phi_{s^{\prime}}} \sim C(L) \epsilon^{-1 / 2} \exp (-2(\Delta \mathcal{H})(L) / \epsilon), & & \epsilon \rightarrow 0, \\
C(L) \sim \text { const } \times L, & & L \rightarrow \infty,
\end{array}
$$

where $2(\Delta \mathcal{H})(L)$ is the energy barrier, i.e., the energy of formation of the critical droplet within the spatial domain $[0, L]$. This will tend to a limiting value as $L \rightarrow \infty$, since the droplet tends to a limiting shape. We stress that the asymptotic linear growth of the prefactor with $L$ in this case occurs for a simple physical reason: if periodic boundary conditions are imposed, the critical droplet may form with equal likelihood anywhere in the spatial domain. Departures from linearity will occur when $L$ is small, but they are due to the droplet assuming its limiting shape only in the large- $L$ limit. The large- $L$ behavior of the prefactor displayed in (13) is well-known, but the finite-volume case of fixed $L$ has only recently been systematically treated [15].

\section{STOCHASTIC MICROMAGNETISM}

We can now relate the low-temperature micromagnetic reversal rate theories of Brown [3] and Braun [4, 5, 6, 7, 8] to finite-dimensional and infinite-dimensional Kramers theory, respectively, and explain the physical origin of the $T^{-1 / 2}$ prefactor that modifies each of their Arrhenius laws. Our discussion will indicate how Braun's treatment of a very long $(L \rightarrow \infty)$ ferromagnetic nanowire can be modified and extended to the case of a thin ferromagnetic cylinder with flat ends, and fixed length $L$.

The starting point for continuum micromagnetic modeling [1] is the Landau-Lifshitz-Gilbert equation for the bulk magnetization vector $\mathbf{m}$. If dimensionless units are used and the magnetization unit is chosen so that the saturation magnetization of the ferromagnetic material being modeled equals unity, i.e., so that $|\mathbf{m}|=1$ at each point in the sample, this equation is

$$
\dot{\mathbf{m}}=-\gamma \mathbf{m} \times \mathbf{h}_{\mathrm{eff}}+\alpha \mathbf{m} \times \dot{\mathbf{m}},
$$

where $\gamma>0$ is the gyromagnetic ratio. The first term causes $\mathbf{m}$ to precess about the local magnetic field $\mathbf{h}_{\text {eff }}$. The second is a phenomenological damping term introduced by Gilbert [27, which causes $\mathbf{m}$ to relax toward the direction of $\mathbf{h}_{\text {eff }}$. This becomes clear if the equation is rewritten as

$$
\left(1+\alpha^{2}\right) \gamma^{-1} \dot{\mathbf{m}}=-\mathbf{m} \times \mathbf{h}_{\mathrm{eff}}-\alpha \mathbf{m} \times\left(\mathbf{m} \times \mathbf{h}_{\mathrm{eff}}\right),
$$

which follows by evaluating the cross product of $\mathbf{m}$ with each term. The Gilbert damping term, which is now universally used as a replacement for a less satisfactory damping term proposed earlier by Landau and Lifshitz [28], is still slightly controversial, in part because of the difficulty of calculating the material-dependent dimensionless damping coefficient $\alpha>0$ from first principles.

The formula for $\mathbf{h}_{\text {eff }}$ must capture much of the physics. If the dynamics are essentially Hamiltonian (with the exception of the Gilbert damping), then $\mathbf{h}_{\text {eff }}=-\delta H[\mathbf{m}] / \delta \mathbf{m}$ for some Hamiltonian functional $H[\mathbf{m}]$ of the 
magnetic field configuration. The Hamiltonian employed by Braun for a thin cylindrical particle whose axis extends from $x=0$ to $x=L$, with $\mathbf{m}$ taken to depend only on $x$, is

$$
\mathcal{H}[\mathbf{m}]=\int_{0}^{L}\left\{\left[\left(\partial_{x} m_{x}\right)^{2}+\left(\partial_{x} m_{y}\right)^{2}+\left(\partial_{x} m_{z}\right)^{2}\right]+(\mathbf{m}, \mathrm{K} \mathbf{m})-\mathbf{h}_{\mathrm{ext}} \cdot \mathbf{m}\right\} d x .
$$

The first term is the exchange term, of quantum-mechanical origin, which favors the alignment of nearby magnetic moments, and the second is a quadratic form on the sphere $S^{2} \ni \mathbf{m}$ that models the anisotropy of the material, if any. For convenience the unit of length here is chosen to equal the exchange length, so the coefficient of the exchange term is unity.

This Hamiltonian does not include a term of a type arising from classical electromagnetism, the omission of which has been criticized by Aharoni [11, 12. If $\boldsymbol{\nabla} \cdot \mathbf{m} \neq 0$ in the interior of the sample then there will be a magnetostatic volume charge, which will generate its own magnetic field, with which the bulk magnetization will interact. The additional field is called the 'demagnetizing' field, for an obvious reason. In general, this $\mathbf{m}$-generated field will add a term to the energy density which is nonlocal in space. However, as mentioned in Section 1] Braun [14 has given convincing arguments for replacing this term in the thin-cylinder limit by a local term, which can be absorbed into a renormalization of the anisotropy tensor $\mathrm{K}$.

Braun considered the effects on the Landau-Lifshitz-Gilbert equation (15) of adding a small random term of the form $\epsilon^{1 / 2} \boldsymbol{\eta}(x, t)$ to $\mathbf{h}_{\text {eff }}$, where $\boldsymbol{\eta}(x, t)$ is vectorial spatiotemporal white noise. Before discussing his results, it is useful to review Brown's much earlier theoretical treatment of ferromagnetic reversal in a single-domain particle. The equation (15) makes sense as the evolution equation of a single vector $\mathbf{m}$, regarded as the spatially uniform saturation magnetization across such a particle. The appropriate Hamiltonian is not (16) but rather

$$
\mathcal{H}[\mathbf{m}]=(\mathbf{m}, \mathrm{Km})-\mathbf{h}_{\mathrm{ext}} \cdot \mathbf{m},
$$

so

$$
\mathbf{h}_{\text {eff }}=-\delta \mathcal{H}[\mathbf{m}] / \delta \mathbf{m}=\mathbf{h}_{\text {ext }}-2 \mathrm{~K} \mathbf{m} .
$$

The anisotropy tensor $\mathrm{K}$ should include both shape and crystalline anisotropies. Brown considered the case of uniaxial anisotropy, with the external field directed along the 'easy axis' of the particle. If this easy axis points in the $x$-direction, $\mathrm{K}=\operatorname{diag}\left(-K_{e}, 0,0\right)$ for some $K_{e}>0$, so let $\mathbf{h}_{\text {ext }}=\left(h_{\text {ext }}, 0,0\right)$ with $h_{\text {ext }}>0$. Then as a function of the angle $\theta$ between $\mathbf{m}$ and the $x$-axis,

$$
\mathcal{H}[\mathbf{m}]=-K_{e} \cos ^{2} \theta-h_{\text {ext }} \cos \theta .
$$

Provided that $h_{\text {ext }}<2 K_{e}$, this function on the sphere $S^{2} \ni \mathbf{m}$ has two minima: the metastable state $\theta=\pi$ (antiparallel to $\mathbf{h}_{\text {ext }}$ ) and the stable state $\theta=0$ (parallel to $\mathbf{h}_{\text {ext }}$ ), which has lower energy. Their energies are respectively $-K_{e} \pm h_{\text {ext }}$.

It is easy to check that according to the evolution equation (15), the particle magnetization $\mathbf{m}$ will spiral into one or the other of these two states. On the sphere, the separatrix between their domains of attraction is the parallel of latitude singled out by the equation $\theta=\cos ^{-1}\left(-h_{\mathrm{ext}} / 2 K_{e}\right)$. This is an unstable limit cycle. If a temporal noise term of the form $\epsilon^{1 / 2} \boldsymbol{\eta}(t)$, with $\epsilon \ll 1$, is added to $\mathbf{h}_{\text {eff }}$, then the noise will eventually drive the magnetization vector away from the metastable state and across this separatrix. It can be shown that the optimal trajectory for noise-activated escape is, in fact, a time-reversed infalling spiral. So the mechanism of activated magnetization reversal in this model is of the sort discussed in Section 2 However, it should be noted that when $h_{\text {ext }}>2 K_{e}$, the metastable state becomes unstable, and there is no separatrix to be crossed. Reversal in the presence of a sufficiently high external field is field-activated rather than noise-activated.

By examination, the 'height' of the unstable limit cycle in terms of energy is $h_{\text {ext }}^{2} / 4 K_{e}$, so the energy barrier to reversal is $2 \Delta \mathcal{H}=2\left[h_{\text {ext }}^{2} / 4 K_{e}-\left(-K_{e}+h_{\text {ext }}\right)\right]$. The weak-noise reversal rate will be given by (4), i.e.,

$$
\Gamma \sim \text { const } \times \epsilon^{-1 / 2} \exp (-2 \Delta \mathcal{H} / \epsilon), \quad \epsilon \rightarrow 0 .
$$

The factor 'const' can be calculated by integrating along the limit cycle the rate at which the probability density of $\mathbf{m}$ is absorbed. However, this derivation already makes it clear why the Néel-Brown reversal rate, as it is now 
called, falls off in the limit of low temperature in a non-Arrhenius way. The anomalous power $\epsilon^{-1 / 2}$, i.e., $T^{-1 / 2}$, arises from the need to integrate a probability flux around the entire limit cycle, rather than over an $O\left(\epsilon^{1 / 2}\right)$ transverse lengthscale near a saddle point, as is more typical in the Kramers theory of noise-activated escape. This derivation can be extended to the case of a single-domain ferromagnetic particle with mildly non-uniaxial anisotropy tensor $\mathrm{K}$, in which, as here, the barrier to reversal is an unstable limit cycle.

We now turn to the thin nanowire computations of Braun. In their full generality, they apply to a thin nanowire of a ferromagnetic material with cubic anisotropy, the easy axis of which is aligned with the axis of the wire; which may be taken to be the $x$-axis. By adding an appropriate multiple of the identity matrix to $\mathrm{K}$ (which has no physical effect, since $|\mathbf{m}|=1$ at all points in the sample), and rotating the coordinate system about the wire, $\mathrm{K}$ can be taken to equal diag $\left(-K_{e}, 0, K_{h}\right)$ for some $K_{e}, K_{h}>0$. The $z$-axis will be a hard axis, and the $x-y$ plane an easy plane, within which the magnetization vector at every point along the wire is preferentially confined. A hard axis of this sort could arise from a shape anisotropy rather than a crystalline anisotropy, e.g., from the cross section of the wire being elliptical rather than circular.

For simplicity we consider here only the limiting case $K_{h} \rightarrow \infty$, in which all fluctuational motion of the magnetization vector out of the $x-y$ plane is suppressed. (Taking out-of-easy-plane fluctuations into account would alter the Kramers prefactor by multiplying it by an extra determinant quotient, but would not affect the qualitative nature of its $\epsilon \rightarrow 0$ and $L \rightarrow \infty$ asymptotics.) For this case the effective Hamiltonian is

$$
\mathcal{H}[\mathbf{m}]=\int_{0}^{L}\left\{\left[\left(\partial_{x} m_{x}\right)^{2}+\left(\partial_{x} m_{y}\right)^{2}\right]-K_{e} m_{x}^{2}-h_{\mathrm{ext}} m_{x}\right\} d x .
$$

As a functional of the angle $\theta$ between $\mathbf{m}$ (in the $x-y$ plane) and the $x$-axis,

$$
\mathcal{H}[\theta]=\int_{0}^{L}\left[\left(\frac{d \theta}{d x}\right)^{2}-K_{e} \cos ^{2} \theta-h_{\text {ext }} \cos \theta\right] d x .
$$

In the absence of thermal noise, the stationary configurations of the deterministic Landau-Lifshitz-Gilbert equation (15) will be those for which $\delta H[\mathbf{m}] / \delta \mathbf{m}=0$, i.e., $\delta H[\theta] / \delta \theta=0$. That is, they will be those for which the magnetization inclination angle $\theta$ as a function of distance $x$ along the wire satisfies

$$
2 \frac{d^{2} \theta}{d x^{2}}+2 K_{e} \sin \theta \cos \theta+h_{\mathrm{ext}} \sin \theta=0 .
$$

For the moment, suppose that the wire can be viewed in some sense as toroidal, i.e., suppose that periodic boundary conditions $\theta(0)=\theta(L), \theta^{\prime}(0)=\theta^{\prime}(L)$ can be imposed. Then the solutions of (23) will include $\theta \equiv \pi$ and $\theta \equiv 0$, which are the familiar states of metastable and stable magnetization, with the magnetization pointing against or along the external field, which is itself applied parallel to the wire. The solution $\theta \equiv \cos ^{-1}\left(-h_{\text {ext }} / 2 K_{e}\right)$ is also stationary in time, though unstable. This is the uniform (Néel-Brown) barrier to magnetization reversal, which in this context is a saddle point in the infinite-dimensional space of field configurations.

However, if $L$ is sufficiently large, there will also be a nonuniform barrier to magnetization reversal. By proceeding as in our analysis [15] of the Ginzburg-Landau equation (10), one can show that

$$
\theta=\theta_{u}(x) \equiv \cos ^{-1}\left(\frac{a_{1} \operatorname{sn}^{2}\left(\left(x-x_{0}\right) / c \mid m\right)+a_{0}}{b_{1} \operatorname{sn}^{2}\left(\left(x-x_{0}\right) / c \mid m\right)+b_{0}}\right),
$$

where $a_{0}, a_{1}, b_{0}, b_{1}, c, m$ depend on $L$ and $h_{\text {ext }} / 2 K_{e}$, and $x_{0}$ is arbitrary, is also an unstable stationary solution, and is at lower energy than the Néel-Brown barrier. Here $\operatorname{sn}(\cdot \mid m)$ is the Jacobi elliptic function with modular parameter $m \in(0,1)$, the period of which is $4 K(m)$, where $K(m)$ is the first elliptic integral [16]. The parameter $m$ is of course chosen so that $\theta_{u}$, as a function of $x$, has period $L$. If such a lower-energy barrier to reversal exists, its 'height' in terms of energy, which may be written as $\Delta \mathcal{H}(L)$, will determine the exponential falloff of the rate of escape from the metastable state $\theta \equiv \pi$, as the noise strength tends to zero.

At this point, we have gone significantly beyond the analysis of Braun. It is not difficult to check that the solution $\theta=\theta_{u}(x)$ can be viewed as a critical droplet: for many choices of parameter, it resembles a droplet of the 
$\theta=0$ phase inserted into a background consisting of the $\theta=\pi$ phase, and delimited by a $\pi$ wall and a $-\pi$ wall. However, its shape depends on the length $L$ of the wire. As $L \rightarrow \infty$, it tends to a limiting shape, which may be expressed in terms of hyperbolic trigonometric functions. This is possible because as $L \rightarrow \infty$, the modular parameter $m$ tends to unity, and $\operatorname{sn}(x \mid m)$ degenerates to $\tanh x$. The formula (24) is new in this context, though the limiting shape as $L \rightarrow \infty$ was obtained by Braun. Similar expressions for periodic micromagnetic structures, involving elliptic functions, have been derived in other contexts [29].

It follows immediately from the discussion in Section 2 that for this model of a thin cylindrical particle of length $L$ with periodic boundary conditions, the magnetization reversal rate $\Gamma$ satisfies

$$
\begin{array}{ll}
\Gamma \sim C(L) \epsilon^{-1 / 2} \exp (-2(\Delta \mathcal{H})(L) / \epsilon), & \epsilon \rightarrow 0, \\
C(L) \sim \text { const } \times L, & L \rightarrow \infty .
\end{array}
$$

The factor $\epsilon^{1 / 2}$ is due to the fact that the separatrix between the metastable and stable domains of attraction contains a limit cycle of length $L$, since the droplet may be formed anywhere along the wire, on account of translation invariance. ( $x_{0}$ above is arbitrary.) This is also responsible for the asymptotic linear growth of the prefactor with $L$. These two features were noted by Braun, but we now see they have a simple physical origin.

The present approach, in which the shape of the critical droplet for fixed $L$ is precisely specified in terms of elliptic functions, yields precise values for the exponent $2 \Delta \mathcal{H}(L)$ and the prefactor $C(L)$. Details of the calculations, which are numerical rather than analytic, may appear elsewhere. What is of greater interest, however, is the modification of this analysis to include more realistic boundary conditions. Braun has pointed out that in a cylinder of finite length, 'open' boundary conditions with the magnetization perpendicular to the ends may be appropriate [14]. In the present scheme this corresponds to taking $\theta^{\prime}(0)=\theta^{\prime}(L)=0$, i.e., to Neumann boundary conditions.

Equipped with Neumann boundary conditions, the Euler-Lagrange equation (23) still has the metastable and stable states $\theta \equiv \pi, \theta \equiv 0$ as solutions. A detailed analysis, which will be published elsewhere, reveals that by choosing parameters appropriately, the unstable elliptic solution $\theta=\theta_{u}(x)$ of (24) can also be made to satisfy Neumann boundary conditions. However, when this is done, it becomes a 'half-droplet' delimited by a $\pi$ wall and either $x=0$ or $x=L$. Unlike the case of periodic boundary conditions, nucleation at low temperatures is therefore not likely to take place anywhere along the wire, but must, on energetic grounds, preferentially begin at either end. Once a critical half-droplet has formed near either end, it may roll 'downhill' in the magnetization configuration space by spreading along the wire, replacing the original value $\theta=\pi$ by $\theta=0$ as it goes.

The important point is that if Neumann rather than periodic boundary conditions are imposed, there will be no infinite degeneracy of the transition state, no unstable limit cycle, no non-Arrhenius $\mathrm{O}\left(\epsilon^{-1 / 2}\right)$ behavior of the Kramers prefactor, and also no asymptotic linear dependence of the prefactor on $L$ as $L \rightarrow \infty$. The noiseactivated magnetization reversal of a long thin ferromagnetic cylinder actually fits much better into traditional Kramers theory if Neumann boundary conditions are imposed.

\section{ACKNOWLEDGMENTS}

This research was supported in part by National Science Foundation Grant No. PHY-0099484.

\section{REFERENCES}

[1] J. Fidler and T. Schrefl, "Micromagnetic modeling - the current state of the art," J. Phys. D 33, pp. R135R156, 2000.

[2] A. Aharoni, Introduction to the Theory of Ferromagnetism, Oxford University Press, Oxford, 2nd ed., 2000.

[3] W. F. Brown, Jr., "Thermal fluctuations of a single-domain particle," Phys. Rev. 130(5), pp. 1677-1686, 1963.

[4] H.-B. Braun, "Thermally activated magnetization reversal in elongated ferromagnetic particles," Phys. Rev. Lett. 71(21), pp. 3557-3560, 1993. 
[5] H.-B. Braun, "Fluctuations and instabilities of ferromagnetic domain-wall pairs in an external magnetic field," Phys. Rev. B 50(22), pp. 16485-16500, 1994.

[6] H.-B. Braun, "Statistical mechanics of nonuniform magnetization reversal," Phys. Rev. B 50(22), pp. 16501$16521,1994$.

[7] H.-B. Braun and H. N. Bertram, "Nonuniform switching of single domain particles at finite temperatures," J. Appl. Phys. 75(9), pp. 4609-4616, 1994.

[8] H.-B. Braun, "Kramers' rate theory, broken symmetries, and magnetization reversal," J. Appl. Phys. 76(10), pp. 6310-6315, 1994.

[9] L. S. Schulman, Techniques and Applications of Path Integration, ch. 29. Wiley, New York, 1981.

[10] E. D. Boerner and H. N. Bertram, "Non-Arrhenius behavior in single domain particles," IEEE Trans. Magnetics 34(4), pp. 1678-1680, 1998.

[11] A. Aharoni, "Magnetostatic energy of the soliton in a cylinder," J. Magnetism and Magnetic Materials 140-144, pp. 1819-1820, 1995.

[12] A. Aharoni, "Comment on "Kramers' rate theory, broken symmetries, and magnetization reversal"," J. Appl. Phys. 80(5), pp. 3133-3134, 1996.

[13] J. S. Broz, H.-B. Braun, O. Brodbeck, W. Baltensperger, and J. S. Helman, "Nucleation of magnetization reversal via creation of pairs of Bloch walls," Phys. Rev. Lett. 65(6), pp. 787-789, 1990.

[14] H.-B. Braun, "Nucleation in ferromagnetic nanowires - magnetostatics and topology," J. Appl. Phys. 85(8), pp. $6172-6174,1999$.

[15] R. S. Maier and D. L. Stein, "Droplet nucleation and domain wall motion in a bounded interval," Phys. Rev. Lett. 87(27), paper no. 270601, 2001. Available as arXiv:cond-mat/0108217.

[16] M. Abramowitz and I. A. Stegun, eds., Handbook of Mathematical Functions, Dover, New York, 1965.

[17] W. F. Brown, Jr., Micromagnetics, no. 18 in Interscience Tracts on Physics and Astronomy, Interscience, New York, 1963.

[18] A. Aharoni, "Reduction in coercive force caused by a certain type of imperfection," Phys. Rev. 119(1), pp. 127-131, 1960.

[19] G. Brown, M. A. Novotny, and P. A. Rikvold, "Micromagnetic simulations of thermally activated magnetization reversal of nanoscale magnets," J. Appl. Phys. 87(9), pp. 4792-4794, 2000. Available as arXiv:cond-mat/9909136

[20] G. Brown, M. A. Novotny, and P. A. Rikvold, "Langevin simulation of thermally activated magnetization reversal in nanoscale pillars," Phys. Rev. B 64, paper no. 134422, 2001. Available as arXiv:cond-mat/0101477

[21] P. Hänggi, P. Talkner, and M. Borkovec, "Reaction-rate theory: Fifty years after Kramers," Rev. Modern Phys. 62(2), pp. 251-341, 1990.

[22] H. A. Kramers, "Brownian motion on a field of force and the diffusion model of chemical reactions," Physica 7(4), pp. 284-304, 1940.

[23] C. Kipnis and C. M. Newman, "The metastable behavior of infrequently observed, weakly random, onedimensional diffusion processes," SIAM J. Appl. Math. 45(6), pp. 972-982, 1985.

[24] J. S. Langer, "Theory of the condensation point," Ann. Physics 41(1), pp. 108-157, 1967.

[25] J. S. Langer, "Statistical theory of the decay of metastable states," Ann. Physics 54, pp. 258-275, 1969. 
[26] A. J. McKane and M. B. Tarlie, "Regularization of functional determinants using boundary conditions," J. Phys. A 28(23), pp. 6931-6942, 1995. Available as arXiv:cond-mat/9509126

[27] T. L. Gilbert and J. M. Kelly, "Anomalous rotational damping in ferromagnetic sheets," in Proceedings of the 1st Magnetism and Magnetic Materials Conference, AIEE Special Publications (T-78), pp. 253-263, (Pittsburgh), 1955.

[28] S. Iida, "The difference between Gilbert's and Landau-Lifshitz's equations," J. Phys. Chem. Solids 24, pp. $625-630,1963$.

[29] M. J. Shirobokov, "On the mechanism of cobalt magnetization," Dokl. Akad. Nauk SSSR 24(5), pp. 426-429, 1939. 